Copyright (C 2014 IEEE. Personal use of this material is permitted. Permission from IEEE must be obtained for all other uses, in any current or future media, including reprinting/republishing this material for advertising or promotional purposes, creating new collective works, for resale or redistribution to servers or lists, or reuse of any copyrighted component of this work in other works. 


\title{
Portable Tongue-Supported Human Computer Interaction System Design and Implementation
}

\author{
Rohan Quain and Masood Mehmood Khan
}

\begin{abstract}
Tongue supported human-computer interaction (TSHCI) systems can help critically ill patients interact with both computers and people. These systems can be particularly useful for patients suffering injuries above $C 7$ on their spinal vertebrae. Despite recent successes in their application, several limitations restrict performance of existing TSHCI systems and discourage their use in real life situations. This paper proposes a low-cost, less-intrusive, portable and easy to use design for implementing a TSHCI system. Two applications of the proposed system are reported. Design considerations and performance of the proposed system are also presented.
\end{abstract}

\section{INTRODUCTION}

The tongue has been used for sensory substitution and enabling blind people see and perceive [1-4]. Experiments demonstrated efficacy of the tongue in both tactile and auditory substitution [5]. Critically ill and impaired patients require special purpose communication systems for interacting with the world. Several systems are currently available to such patients. The use of each system is dependent upon the patient-condition and the bodily functions they have control over. Three common communication technologies available for impaired patients are: Eye-activated systems [1-3], Tongue-driven systems [46] and Smart navigation systems [7]. The Eye Writer uses a micro video camera together with infrared light emitting diodes (LED's) to track the light reflected by the eyes dark pupil. The Tongue Drive System requires a permanent magnet to be attached onto the tongue of the patient. The magnet is tracked using an array of Hall Effect sensors located within a dental plate inside the patients mouth. SmartNAV uses infrared light to track the position of a small reflective dot placed upon the user's forehead. As the user moves the position of their head, the location of the dot is tracked using an infrared imaging unit. The output signals of all three systems allow the patient to interact directly with a computer and perform tasks like drawing images and writing documents [1-7].

The eye tracking systems are easy to implement in a human-computer interaction (HCI) system as the long as the

Manuscript received December 20, 2013. This work was partially supported by the Department of Mechanical Engineering, Curtin University of Technology, Perth, Australia.

Rohan Quain is with the Poseidon Program at Raytheon Australia (phone: +61 0419659221; e-mail: rohanquain@gmail.com).

Masood Mehmood Khan is with Curtin University, Department of Mechanical Engineering, Perth Western Australia 6012 (phone: +61 89266 9205; e-mail: Masood.Khan@curtin.edu.au). patient is immobile. However, they cannot be used to navigate electric wheelchairs, as it is not possible for patients to scan ahead to view the surroundings and steer the wheelchair at the same time [4].

The SmartNAV system cannot be used with high level spinal cord patients as they do not have control over their head and neck muscles. Furthermore, the system is not designed to operate electric wheelchairs [7].

Thus, we need a human-computer interaction system that would allow critically impaired patients to navigate both computers and electric wheelchairs. Such interactive systems should be less intrusive than the existing systems and should have the ability and portability to be easily transferred from the patient's bed to a wheelchair. It is expected that such a user friendly and adaptable system will result in increased adoption by the medical industry and provide better comfort for patients.

An overview of the existing TSHCI systems is first presented in this paper. A novel system-architecture is then proposed for designing an improved TSHCI system. Initial implementation of the proposed system is also discussed. Finally, measures and opportunities for improving the TSHCI systems are discussed.

\section{EXISTING TSHCI SYSTEMS}

The use of the tongue as an input mechanism for communication devices was recently investigated at Curtin University [5]. The primary function of the device was to act as an emergency alarm system, notifying attendants and nurses that help is required. This removes the need for constant supervision and care by nursing staff. The implemented Infrared Tongue Activated emergency help Beacon (ITAB), functions using simultaneous and synchronised operation of two sub-systems. A transmitting sub-system that receives input from a patient and generates an infrared signal. The second component, a receiving circuit, receives this signal to activate the hospitals alarm or emergency system. The receiver circuit detects this signal

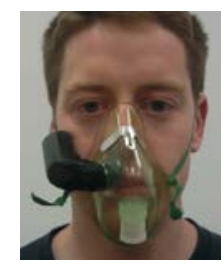

Fig. 1. ITAB mounted on a face 


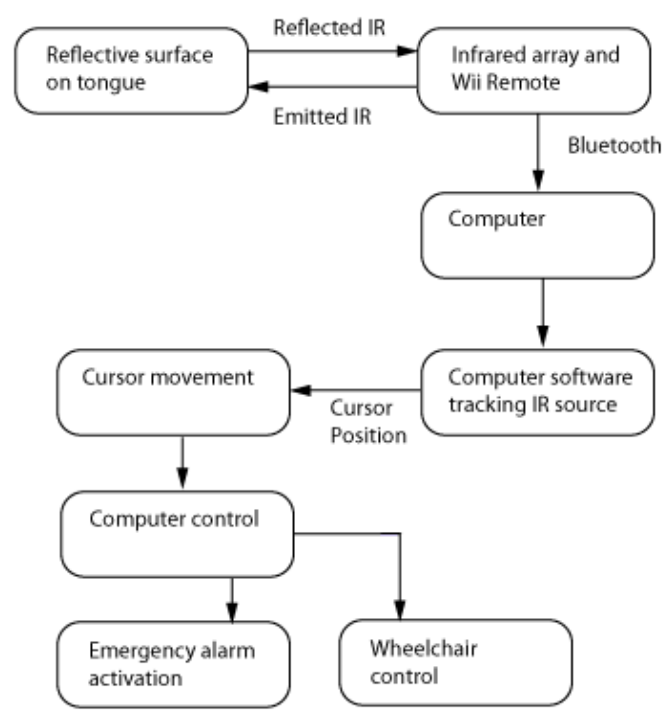

Fig. 2. Proposed system configuration

and activates an alarm. The ITAB, being mounted on a human face, is shown in Fig. 1. As obvious in Fig. 1, the unit is mounted onto an oxygen mask. It achieves its intended purpose of functioning as an emergency device, but it cannot be used for other purposes such as communication and control of an electric wheelchair.

Georgia Tech's Tongue Drive System (TDS) is an important and more versatile TSHCI system [4]. This wireless device enables patients with spinal cord injuries to drive an electrically powered wheelchair and control a computer. For the TDS to work, a small permanent magnet must be attached to the user's tongue through either piercing or gluing. An array of Hall-effect magnetic field sensors located inside the user's mouth is used to detect the change in magnetic field as a result of the tongues movement. These changes are sent to a control unit, from which, the signals are wirelessly transmitted to a Smartphone. The Smartphone converts the signals sent from the sensors into usable commands. These commands can be used to control a wide range of devices such as a computer or wheelchair. The circuitry of the TDS is embedded in a dental retainer. The system is able to interpret seven distinct tongue movements. These intra-oral movements are translated into usable commands. A simple functional depiction of the tongue drive system is available on Georgia Tech's web site.

The TDS can provide some independence; the wheelchair

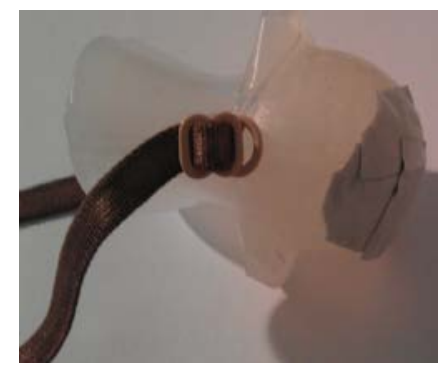

Fig. 3. Silicone tongue device with attached reflective tape

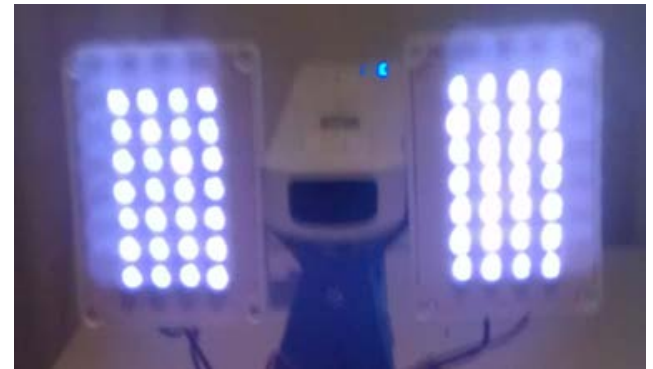

Fig. 4. The Infrared Array System

navigation system has a high degree of control so it can maneuver through shopping isles and crowded streets. The motions required by the tongue are natural and the tongue does not have to apply force for input, this means the chances of muscle fatigue injuries are low. The design has the added advantage of being entirely mounted inside the mouth; this removes any external sign which indicates that the user has a disability. The only drawbacks of the TDS, is the need for the permanent magnet to be eventually pierced onto the tongue and the requirement of having a dentist fit a dental retainer customized for each individuals mouth. This procedure may not be financially and physically possible for all patients. Overall the TDS is a moderately-priced, flexible and easy to operate solution which allows movement and communication for the patient [4].

\section{PRoposed System Design}

In an attempt to avoid the intrusive operation and functional limitations of prevailing TSHCI systems, a design framework presented was developed. This focused upon giving patients the ability to communicate with hospital staff in the case of emergency and to allow control of computers and wheelchairs, whilst ensuring the device was affordable and unobtrusive for the patient. It provided the functional specifications for a future TSHCI system. The TSHCI device generated from the specifications is centered on the principle of tracking infrared light reflected by the tongue with a Nintendo Wii Remote. The proposed system ensured a more acceptable and adoptable TSHCI system. The new TSHCI system's configuration is shown in Fig. 2. Major functional components of the final system are described hereunder.

\section{1) Reflective Surface on Tongue}

For the system to deliver user control and work in a suitable range, a reflective medium was used to reflect infrared light to a Wii remote's infrared camera. [8-9]. The effective range of the tape when used as a reflective medium was found to be $150 \mathrm{~cm}$. The tape also provided accurate feedback positioning. The reflective tape was located on the tongue considering three main issues: medium had to be as unobtrusive as possible, had to be able to be worn for extended periods of time, and had to be easy to clean. The device selected to meet the criteria was a silicone tongue device usually worn by people suffering sleep apnea. The device can be removed by patients simply by bringing their teeth together to break the air seal. The silicone tongue 
device is a low cost solution to the reflective tape mounting. It can be comfortably worn for long periods of time as demonstrated in its use as a sleep assistive device. Cleaning is easy as it is made of a medical grade silicone which can be easily sterilized. The silicone tongue device is shown in Fig. 3.

\section{2) Infrared Array Design}

The requirements of the infrared array design were determined by its application and the specifications of the Wii remote's infrared camera. Firstly, the Tongue tracking system is not likely to be required for use by patients at distances greater than $1.5 \mathrm{~m}$. Secondly, the Wii remote's IR pass filter restricts the camera to detect light sources within the near-infrared spectrum, $800 \mathrm{~nm}$ to $940 \mathrm{~nm}$ in wavelength. The infrared light will need to travel to both; the patient and back to the infrared camera. Therefore a sizeable infrared array is required to output enough light intensity. The selected array, shown in Fig. 4 was a KEMO made, 4 x 7 array. The employed infrared LED's (TSHA6202) have a peak wavelength of $875 \mathrm{~nm}$. This was an ideal wavelength as it falls in the middle of the suitable range of wavelengths for the Wii's infrared camera. The angle of half intensity of the LED is $\pm 12^{\circ}$. This makes the array powerful enough to achieve adequate ranges at wider viewing angles. This solution was found to be cost effective and robust [9].The forward current of the LED was $100 \mathrm{~mA}$, with 4 parallel strings per array arranged to result in a total of 8 strings, each requiring $100 \mathrm{~mA}$. In order to power the array, a $13.5 \mathrm{~V}$ DC supply outputting a total of $800 \mathrm{~mA}$ was used to ensure that the LED's were outputting the required light intensity. A simple bi-directional switch was added in order to turn on and off the connection to the supply.

3) Wii remote

The outputs of the Wii remote were processed in two stages. Firstly a connection between the remote and the computer was made via Bluetooth. Once this was established, the raw infrared data (output) emitted by the remote could be translated into usable outputs by a suitable software program.

4) Wii remote to $P C$ Connection

A Bluetooth dongle with Bluetooth specification V2.1 and Class 1, of $100 \mathrm{~m}$ range (Digitech) was connected to a laptop computer operating on Windows 7. Due to the Wii remote being classed as a Human Interface Device (HID) class peripheral, the Bluetooth software was able to recognize the presence of the remote and connect upon a simple mouse click. Now that a connection was established, a software program is required to handle the inputs of the Wii remotes infrared camera and translate the incoming data into both cursor movement and outputs needed to control any wheelchair type device. The employed software, FreeTrack, a multi-purpose optical motion tracking software, can be used to accurately track sources of infrared light. The main aim of using FreeTrack, was to track the tongue tip movements and give patients control over a mouse cursor, as cursor control opens the door to a variety of communication and transport possibilities for patients. To achieve this goal, FreeTrack was used together with a dwell clicking program in order to provide the mouse clicking functionality.

The mouse cursor navigation task was accomplished by first generating a profile within FreeTrack which could be used for mouse control. Profiles within FreeTrack allow for the system to incorporate the needs of each individual patient; making it simple to quickly adapt to the needs of different people. FreeTrack uses threshold control in order to eliminate unwanted sources of light interference, the threshold level was set by adjusting the threshold control bar until only the infrared blob which was being reflected back by the reflective tape was visible on the FreeTrack blob tracking interface. FreeTrack's center feature was then used to ensure FreeTrack would take the current position of the tongue to be the center position for all future output purposes. Once the threshold level and center position are set, the mouse output, together with the auto pan option can be selected within the program. The auto pan function allows the mouse cursor to move across the entirety of the screen, it works by initially detecting that the tongue is located away from the center point. The mouse cursor will then continue to auto pan in the same direction that the tongue is from the center point, when the tongue is brought back to the center point the cursor will stop. After a set period of time, a dwell clicking program (Dwell Clicker 2) will initiate a mouse click. Dwell clicking programs are commonly used by people suffering from repetitive strain injuries, it provides full functionality for the user in the sense that it can achieve left-click, right-click, double-click or drag. Click options can easily be changed through the use of large usable icons.

Initial tests showed that the distance travelled by the tongue away from the center point was too small for the FreeTrack program to initiate the auto pan feature. In order to achieve the activation of the auto pan feature, the input to output gain ratio was changed within the profile. Overall the FreeTrack software had many useful features which could be harnessed in order to improve the overall functionality of the tongue tracking system.

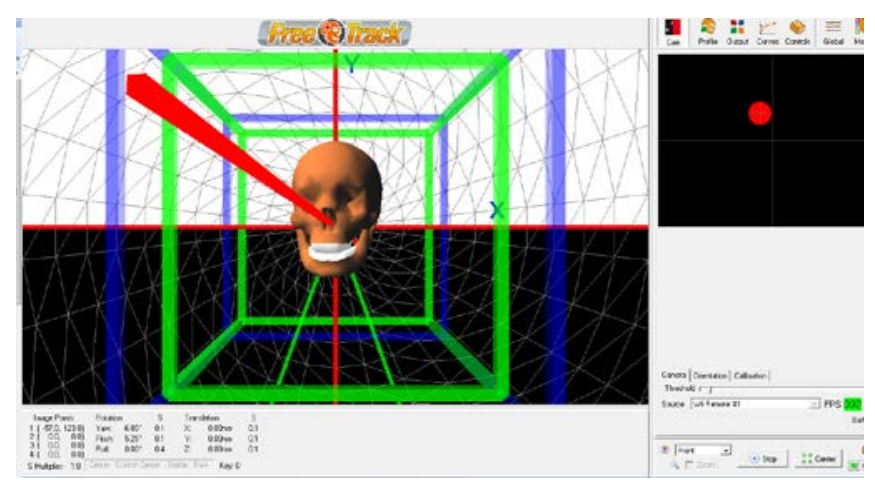

Fig. 5. Freetrack operation showing the movement of an object (offcenter position). Dark red lines intersect at the origin and their extension indicates the horizontal and vertical axes. A dark red vector shows the current position and orientation of the user. Green and purple lines show the 3-dimensional world frames. 


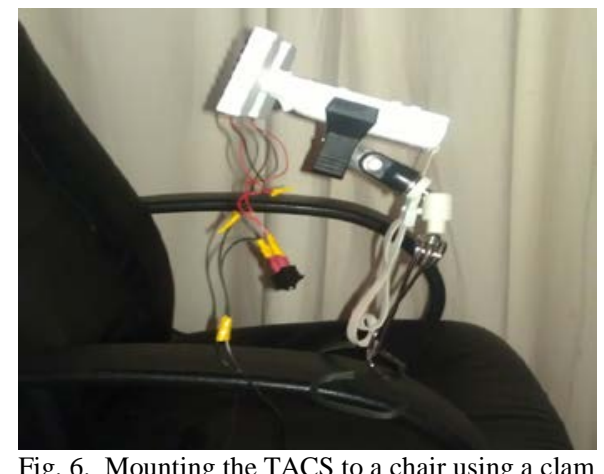

\section{SYSTEM MOUNTING}

The proposed Tongue Activate Control System (TACS) must be mounted well to ensure that the system can remain functional at all times. Critically ill patients primarily spend their time either in a bed or sitting on a wheelchair. The TACS must be able to support transition between these two positions as easily as possible. In order to accomplish this, a flexible mounting system must be developed as patients' room sizes, bed sizes and wheelchair models can differ.

As the Nintendo Wii Remote is a mass produced item, many existing solutions exist. The Deluxe Wii Remote Mount kit was selected for use in this project as it provides a $180^{\circ}$ tilt range includes mounting options for ceiling and table use. It also uses standard 1/4 inch threads throughout, allowing for the mounting to be modified easily to suit.

\section{A. Electric Wheelchair Operation}

The TACS can be easily adjusted for any electric wheelchair. A carer can make the necessary adaptions in less than a minute. For wheelchair use the Wii's mounting base plate can be disconnected and replaced with a clamp which screws into the main shaft, as shown in Fig. 6. The infrared array requires an input voltage of $12-14 \mathrm{~V}$ DC for optimum operation, meaning that the array can be connected directly to the output terminals of the standard $12 \mathrm{~V}$ DC battery used for the electric wheelchair. A small netbook computer running the FreeTrack program can be permanently attached to the wheelchair, no wires will need to be connected as the Wii remote connects to the PC using Bluetooth.

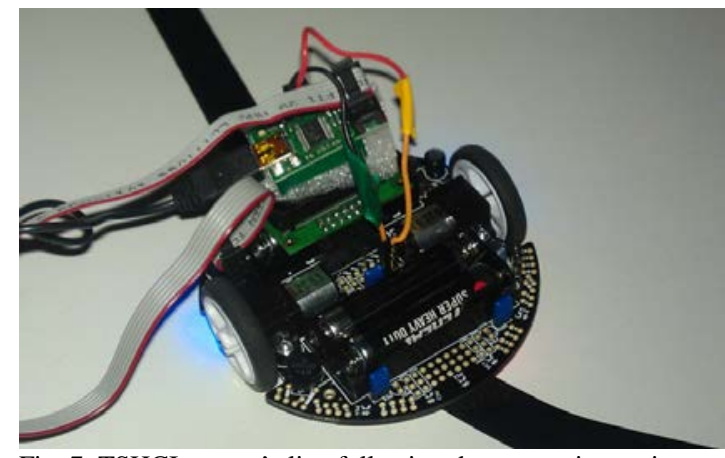

Fig. 7. TSHCI system's line following demonstration action

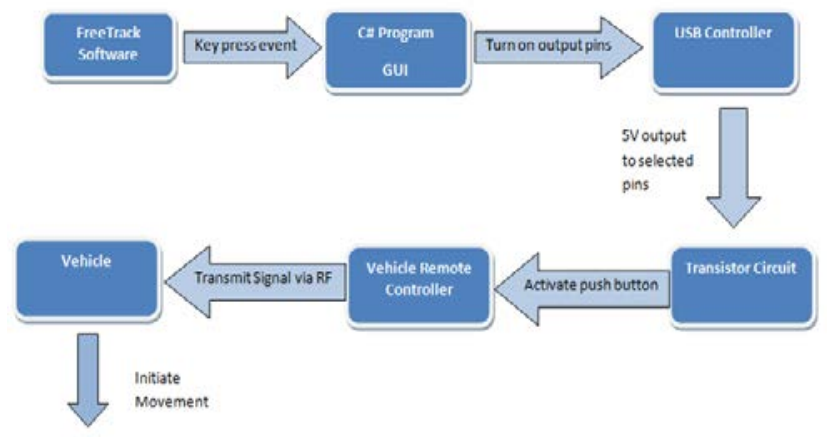

Fig. 8. The navigation schema for operating a remotely controlled car

\section{B. Remotely Controlled Navigation}

A DIGITECH Mini High Speed remotely controlled $(\mathrm{R} / \mathrm{C})$ car was also operated with the system. The output from a mouse control signal was replaced by the FreeTrack program signals to trigger the key press events. For example, when the tongue's position is to the right of center, a " $\mathrm{D}$ " key press event was outputted. The employed navigation scheme is shown in Fig. 8 demonstrating how the software is configured in order to achieve the appropriate output signal. It is important to note that while using the Tongue device with FreeTrack, only one infrared blob could be used by the program. So it was being operated in single point mode. In single point mode translation along the $\mathrm{x}$ and $\mathrm{y}$ axis is detected in the program as yaw and pitch movements respectively. Therefore FreeTrack can output a total of four usable signals, yaw left/right and pitch up/down.

We developed a software program in C-Sharp (C\#) to serve two purposes. Firstly, for recognizing the 'key-press event' and for transmitting an appropriate signal to the vehicle. Secondly, for serving as a training tool for patients as they begin to become familiar with moving their tongue to control a simple vehicle.

When a key press event is generated by FreeTrack from one of the four possible output movements by the tongue, the program recognizes the key pressed and sets the necessary output pins on the USB controller to high. A total of five navigation states are possible for the small vehicle: Veer to the left, Veer to the right, forward, reverse and stop. In order to enter the reverse state the user must first lower their tongue to enter the stop state and then again to enter reverse mode. An example of common operation: if the patients tongue moves to the right, the ' $\mathrm{D}$ ' key will be pressed by FreeTrack, the C\# vehicle control program will detect the ' $\mathrm{D}$ ' key has been pressed and set the forward and right pin to high on the USB Controller. The current state of the program is then shown for learning purposes through colorful directional arrows on a graphical user interface. 


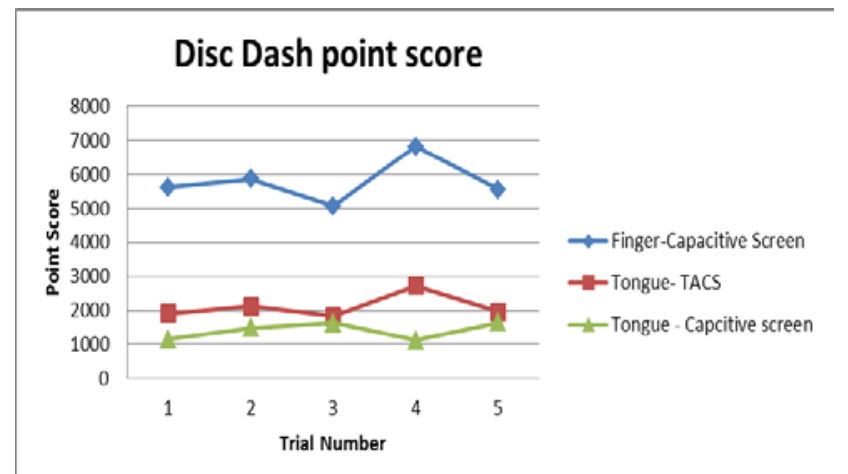

Fig. 9. Disc Dash point scores for the TACS and the initial capacitive screen system.

\section{TEST APPLICATION}

The TACS successfully demonstrated the ability to activate an Emergency Alarm and has proven its potential use for the control of an electric wheelchair. A local alarm buzzer together with an internal hospital alarm system was shown to be triggered through using the TACS to click on an emergency icon on the computer. The capability of the TACS to control an electric wheelchair was achieved by firstly using the system to change the direction of a simple remote control car. An improved level of control was then obtained by interaction of the tongue and a 3pi robot (Fig. 7). The TACS possessed the ability to proportionally control the speed of the robot and to also switch to a line following mode. A patient could use this mode to traverse within their house or large hospitals with ease.

\section{RESUlts}

The necessary performance criteria of the system were established through an extensive literature review. The performance of a system designed to help ill patients communicate can be measured in three key areas:

TABLE I System Performance Results

\begin{tabular}{|c|c|}
\hline TESTING CRITERIA & Result/s \\
\hline \multicolumn{2}{|c|}{ System Operation } \\
\hline Range of Operation & $0.08 \mathrm{~m}$ (minimum) to $5 \mathrm{~m}$ (maximum) \\
\hline \multicolumn{2}{|r|}{ Effectiveness } \\
\hline Typing & $6.2 \mathrm{wpm}$ \\
\hline Mouse control & $\begin{array}{l}2112.8 \text { average point score } \\
33.2 \% \text { improvement }\end{array}$ \\
\hline Play games & Yes \\
\hline \multicolumn{2}{|c|}{ Functionality } \\
\hline Activate Emergency alarm & $\begin{array}{l}\text { Hospital Alarm System } \\
\text { Local Alarm Buzzer }\end{array}$ \\
\hline $\begin{array}{l}\text { Computer } \\
\text { Start programs through icons }\end{array}$ & $\begin{array}{l}\text { Yes } \\
\text { - Yes, as displayed by the ability to } \\
\text { activate emergency alarm button } \\
\text { - Yes, can perform fast and accurate } \\
\text { movements as shown by Disc Dash }\end{array}$ \\
\hline $\begin{array}{l}\text { Potentially control an } \\
\text { electric Wheelchair }\end{array}$ & $\begin{array}{l}\text { Yes } \\
\text { - Navigate Remote Control Car } \\
\text { - Smart Robot-Line Following }\end{array}$ \\
\hline
\end{tabular}

\section{System range of operation}

2. Effectiveness

3. Functionality

The capabilities of the system were first compared against the thumb using an online game called Disc Dash [15]. The game tests mouse movement skills, speed and accuracy. The aim of the game is to click upon the rings as they appear, as fast as possible and close to the center. Points are awarded depending upon how close the click is made to center of the circle and the time which has elapsed. The benefit of performing benchmark tests again with Disc dash is that it allows the performance of the final system to be compared with initial prototypes. Testing was carried out by using FreeTrack's auto pan setting, together with Dwell Clicker 2 set at a dwell time of one second. After an initial practice period of 10 minutes, 5 consecutive results were recorded. The recorded results, shown in red in Fig. 9, suggest that the system outperformed the capacitive screen system. The system results are compared with the capacitive touch screen system.

The overall results of system performance evaluations are presented in Table I. These results suggest that the implemented system can perform well in real life situations.

\section{CONCLUSION}

This work demonstrates that the tongue can be used as a medium of input in human-computer interaction systems. It shows how prevailing software and hardware technologies can help in avoiding use of conventional intrusive components and methods in TSHCI systems. A design framework that allowed development of a portable, nonintrusive, easy to use, and low-cost system was adopted. The resulted system was able to meet the design requirements outlined in the proposed framework. The built-in portability and adoptability of the designed TSHCI system would allow it to be used in various real life situations and conditions.

\section{REFERENCES}

[1] P. Bach-y-Rita, M. E. Tyler, and K. A. Kaczmarek, "Seeing with the Brain,” International Journal of Human-Computer Interaction, vol. 15, no. 2, pp. 285-295, 2003.

[2] M. Tyler, Y. Danilov, and P. Bach-y-Rita, "Closing open-loop control system: Vestbular substitution through the tongue," Journal of Integrative Neuroscience, vol. 2, no. 159, 2003.

[3] P. Bach-y-Rita, K.A. Kaczmarek, M.E. Tyler and J. Garcia-Lara, "Form perception with a 49-point electrotectile stimulus array on the tongue: A technical note,” Journal of Rehabilitation Research and Development, vol. 35, no. 4, 1998.

[4] P. Bach-y-Rita, "Physiological considerations in sensory enhancement and substitution,” Europa Medicophysica, Vol. 25, pp. 107-128, 1989.

[5] P. Bach-y-Rita and S.W. Kercel, "Sensory substitution and the human-machine interface," Trends in Cognitive Science, Vol. 7, no. 12, 2003.

[6] E. W. Systems, "Eye Writer 1.0," 2012.

[7] E. Systems, "Eyegaze Edge TM," in Eyegaze Systems, L. Technologies, 2010.

[8] R. Bates and H. O. Istance, "Why are eye mice unpopular? A detailed comparison of head and eye controlled assistive technology pointing 
devices," Universal Access in the Information Society, vol. 2, pp. 280 $-2902003$.

[9] P. Hangue, K. Jeonghee, and M. Ghovanloo, "Intraoral Tongue Drive System Demonstration," in 2012 Biomedical Circuits and Systems Conference (BioCAS), Taiwan, 2012.

[10] S. Sandy and M. M. Khan, "A Tongue-Activated Emergency Beacon for Immobile Patients," in IEEE-EMBS International Conference on Biomedical and Health Informatics (BHI 2012), Shenzhen, China, 2012.

[11] L. N. S. A. Strujic, "An Inductive Tongue Computer Interface for Control of Computers and assistive Devices," IEEE Transactions on Biomedical Engineering, vol. 53, 2006.

[12] NaturalPoint, "SmartNav," 2012 NaturalPoint, https://www.naturalpoint.com/smartnav/ 2012, last accessed 20 February 2014.

[13] J. Lee, "Tracking fingers with the Wii Remote," Y. Tube, Ed., ed: You Tube, 2007.

[14] P. Wronski, "Design and Implementation of a Hand Tracking Interface using Nintendo Wii Remote," MSc, University of Cape Town, Cape Town, 2008.

[15] D. Chandisingh, Disc Dash [Computer Game] Available at http://www.bigfatarcade.com/swf/discdash.swf, 2004. Last accessed in July 5, 2012. 